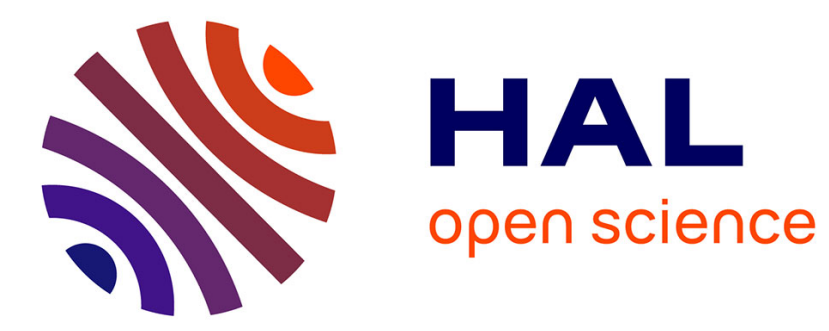

\title{
Retraites : les faux-semblants d'un mouvement social
}

Dominique Andolfatto, Dominique Labbé

\section{To cite this version:}

Dominique Andolfatto, Dominique Labbé. Retraites: les faux-semblants d'un mouvement social. Le Debat, 2011, 163, pp.72-80. hal-00812720

\section{HAL Id: hal-00812720 \\ https://hal.science/hal-00812720}

Submitted on 12 Apr 2013

HAL is a multi-disciplinary open access archive for the deposit and dissemination of scientific research documents, whether they are published or not. The documents may come from teaching and research institutions in France or abroad, or from public or private research centers.
L'archive ouverte pluridisciplinaire HAL, est destinée au dépôt et à la diffusion de documents scientifiques de niveau recherche, publiés ou non, émanant des établissements d'enseignement et de recherche français ou étrangers, des laboratoires publics ou privés. 
Dominique Andolfatto

Université de Bourgogne

(Dominique.Andolfatto@u-bourgogne.fr)

Dominique Labbé

PACTE (IEP de Grenoble)

(dominique.labbe@iep-grenoble.fr)

\section{Retraites : les faux-semblants d'un mouvement social}

(Le Débat, $\mathrm{n}^{\circ}$ 163, janvier-février 2011, p 72-80.)

Résumé

En France, l'automne 2010 a été marqué par d'importantes manifestations et quelques grèves qui n'ont pas réussi à empêcher la réforme des retraites des salariés. Cette impuissance s'explique d'abord par le déclin de la grève et par la désyndicalisation massive. Elle trouve également son origine dans les aides patronales et publiques qui constituent aujourd'hui les ressources principales des syndicats, ce qui les condamne à l'impuissance.

\section{Abstract}

In France, the fall of 2010 was characterized by important social events and some strikes which have failed to prevent the reform of the pension system. This impotence can be explained by the decline of the strike and the large de-unionization. It is also due to subsidies that employers bring to the unions. These subsidies are now the main resources of the unions, that condemned them to impotence.

Le texte ci-dessous a été soumis à la revue Le Débat qui a demandé quelques modifications de pure forme et a assuré la mise en page. Toute citation doit être faite à partir de la revue. 
La réforme des retraites a constitué un long feuilleton au fil de l'année 2010 et a fourni une nouvelle illustration du "déclin du syndicalisme à française" ${ }^{1}$. En effet, les différents épisodes ont mis en lumière quelques aspects caractéristiques de notre pays : réformes en trompe-l'œil, déclin de la grève, faiblesse des syndicats, connivence entre ceux-ci, les employeurs et l'Etat et, pour finir, un curieux soulagement des directions syndicales après un dénouement qui signifiait pourtant leur échec complet.

\section{Une réforme en trompe-l'oeil?}

Cette réforme appartient au registre des nombreuses occasions manquées de la dernière décennie, spécialement du quinquennat Sarkozy. Depuis longtemps, on discute de la réforme des retraites, on convoque élites et experts, on crée des institutions, puis le pouvoir tranche autoritairement. La durée de cotisation des salariés du secteur privé a été allongée (1993), puis celle des fonctionnaires (2003), puis les régimes spéciaux de retraite ont été partiellement remis en cause (2007). Cette fois, on s'est focalisé sur l'âge légal du départ à la retraite - qui est une limite théorique - et pas sur l'âge effectif de l'arrêt d'activité qui est le seul paramètre à prendre en compte pour toute discussion pratique. Or, en France, pour ce qui concerne la retraite des salariés du secteur privé, la différence entre la théorie et la pratique est considérable. Personne n'a semblé conscient qu'une proportion très importante des salariés - à qui l'on demande, théoriquement, de prolonger leur activité - sont déjà, en pratique, sortis du marché du travail et que, pour la quasi-totalité d'entre eux, ils en sont sortis contre leur gré. En effet, dans le secteur privé, l'emploi des "seniors" (salariés de plus de 55 ans) dépend de la conjoncture économique, des décisions des entreprises et pas de la volonté des personnes concernées (même en cas de soi-disant "départ volontaire"). Les entreprises (et de nombreuses administrations) profitent de toutes les occasions pour se débarrasser de leurs salariés âgés. D'ailleurs, à peine la loi avait-elle été promulguée que Renault, Thalès puis Sanofi annonçaient leur désir de se séparer de leurs salariés de 58 ans... C'est pourquoi, en France, en 2008, le "taux d'activité" des 55-64 ans - y compris les chômeurs - n'était que de $39 \%$, ce qui est l'un des taux les plus bas d'Europe, contre $71 \%$ en Suède par exemple.

Si rien ne change dans le comportement des employeurs, la loi ne se traduira pas par un allongement de l'activité - sauf pour les fonctionnaires - mais par un gonflement des chômeurs âgés et par une baisse des pensions qui leur seront finalement servies.

En effet, on a oublié que beaucoup de seniors sans emploi n'ont aucun espoir d'en retrouver avant d'atteindre l'âge théorique de leur retraite. Jusqu'à maintenant, ils bénéficiaient de différents dispositifs qui, peu ou prou, équivalaient à une retraite (spécialement l'Allocation Equivalent Retraite pour ceux qui avaient cotisé le nombre de trimestres requis). Tous ces dispositifs - qui sont en voie de réduction depuis 2009 doivent théoriquement disparaître sans que l'on sache, en pratique, par quoi ils seront remplacés.

Par exemple, la loi de réforme des retraites affirme que l'Etat prendra en charge une partie du salaire des plus de 55 ans nouvellement embauchés par les entreprises. En l'absence des décrets d'application, il est impossible d'estimer la portée de ce dispositif

\footnotetext{
${ }^{1}$ Dominique Andolfatto, Dominique Labbé. Toujours moins ! Déclin du syndicalisme à la française, Paris, Gallimard, 2009.
} 
ni son coût. De toute façon, étant donné la conjoncture économique, un nombre significatif d'embauches de chômeurs seniors est du domaine de la théorie...

Il est également prévu que, avant le $1^{\text {er }}$ juillet 2011 , le gouvernement déposera un rapport devant le parlement sur l'effet de la suppression des dispositifs d'aide aux chômeurs âgés et qu'il proposera des mesures de remplacement. En effet, à la veille d'échéances électorales décisives, comment plonger dans la grande pauvreté plusieurs centaines de milliers de personnes ? Naturellement, la tentation sera grande de loger ces aides dans d'autres budgets que celui de l'Etat... Pour boucher le "trou" des retraites, le président en creusera donc plusieurs autres ailleurs. Mais à la différence du sapeur Camember, il saura certainement le dissimuler, au moins jusqu'après l'échéance électorale de 2012.

Ce constat amène deux questions. Comment les salariés du secteur privé pourront-ils effectivement continuer à travailler jusqu'à 62 ans si aucun employeur n'en veut plus dès 55 ans ? Pourquoi l'Etat, le parlement, le patronat et les syndicats ont-ils fait mine d'ignorer cette question ?

C'est donc la méthode de la réforme et l'attitude des syndicats qui interrogent. $\mathrm{P}$. Cahuc et A. Zylberberg ont bien décrit la méthode de la réforme selon N. Sarkozy: 1 ' "étouffement" des interlocuteurs, soumis à la pression de l'agenda et sommés de discuter dans l'urgence, puis la "conciliation" suivie de reculades². Au final, les changements n'ont pas la portée annoncée et, parfois, ils sont coûteux, comme pour la réforme des régimes spéciaux de retraite.

La réforme de 2010 a été aussi conduite dans l'urgence. Mais, contrairement aux épisodes précédents, la "conciliation" n'a pas eu lieu. Dès le début, il a existé un pacte tacite pour ne pas discuter. Chez les syndicats, personne ne voulait se trouver dans la situation de la CFDT qui ne s'est toujours pas relevée de la grave crise interne provoquée par le soutien de sa direction à la réforme de 2003.

Des deux côtés, le calcul était électoral. Depuis la loi d'août 2008, la représentativité doit se conquérir dans les élections professionnelles et aucune des confédérations ne voulait perdre des voix en semblant cautionner une réforme impopulaire. Compte tenu de cette impasse, et en vue de reconquérir son propre électorat, le président a préféré l'unilatéralisme et la mise en scène de son inflexibilité et de sa détermination.

$\mathrm{Au}$ lendemain de l'échec des manifestations, la CFDT a convenu quelle n'avait jamais cherché à négocier cette réforme, ni imaginé que les manifestations suffiraient à faire reculer le pouvoir ${ }^{3}$. Il s'agissait pour les syndicats, comme pour le président, de mettre en scène leur combativité, de reconquérir l'opinion, tout en évitant soigneusement de se laisser entraîner dans des grèves et une épreuve de force.

\section{Image et réalité de la grève en France}

L'image de la France est celle d'un pays fortement gréviste. En fait, la conflictualité, dans le secteur marchand, a fortement reculé depuis les années 1970. En 2008, 2,4\% des entreprises - comptant 10 salariés ou plus - ont déclaré une "grève" (y compris un simple débrayage $)^{4}$. Le seul indicateur fiable est le nombre de journées de travail

\footnotetext{
${ }^{2}$ Pierre Cahuc, André Zylberberg, Les réformes ratées du président Sarkozy, Paris, Flammarion, 2009, p. 13-14.

${ }^{3}$ Les Echos, 23 novembre 2010.

${ }^{4}$ Myriam Bobbio, Antoine Naboulet, "Négociation collective et grèves dans les entreprises du secteur marchand en 2008", Dares Analyses [ministère du Travail], n 33, juin 2010.
} 
perdues pour grève pour 1000 salariés (tableau ci-dessous). Pour rendre ces données intelligibles, on peut les convertir en probabilité, pour un salarié, de faire une journée de grève au cours de sa vie au travail (dernière colonne).

La conflictualité dans le secteur marchand en 2008

\begin{tabular}{|l|c|c|}
\hline Secteur & $\begin{array}{c}\text { journées de travail perdues } \\
\text { pour grève (pour 1000 } \\
\text { salariés) }\end{array}$ & $\begin{array}{c}\text { Probabilité pour un salarié } \\
\text { de faire une journée grève } \\
\text { en : }\end{array}$ \\
\hline Industrie & 151 & 7 ans \\
Construction & 22 & 45 ans \\
Commerce & 28 & 36 ans \\
Transports & 404 & 2,5 ans \\
Services & 56 & 18 ans \\
\hline Ensemble & 107 & 10 ans \\
\hline
\end{tabular}

Sources : ministère du travail et nos calculs (dernière colonne).

La moitié des salariés français travaillent dans le «tertiaire marchand » (commerce et services hors transport). Au rythme de l'année 2008, ils feraient grève une journée... tous les 35 ans, c'est-à-dire une fois dans toute leur vie active. Pour l'ensemble du secteur marchand (qui inclut l'industrie et les transports, y compris les entreprises publiques), cette probabilité moyenne est de 1 journée de grève tous les dix ans (en léger recul par rapport à 2006 et 2007). Dans les trois fonctions publiques, cette moyenne est d'environ une journée de grève tous les 5 ans (à peu près stable depuis 2005). La série statistique est trop récente pour permettre des comparaisons de long terme.

Malgré ces chiffres, la France conserve une image de pays gréviste. Cette perception provient des grandes vagues de grèves comme 1920, 1936, 1947, 1968, 1995. Elle découle aussi du souvenir d'une décennie "contestataire" (de la fin des années 1960 aux années 1970) et du maintien d'une conflictualité importante dans les transports publics (quoiqu'en recul par rapport au passé récent). Cette image se perpétue aussi à cause du caractère spectaculaire de certaines actions, blocages ou séquestrations, qui sont parfois montées en épingle par les médias. Enfin, si ce recul de la grève est difficilement admis, c'est qu'il heurte un certain "ouvriérisme universitaire" et qu'il semble contradictoire avec la dégradation objective de la situation des salariés français.

En effet, depuis dix ans et, pour la première fois depuis la Libération, le pouvoir d'achat de $90 \%$ des salariés français stagne ou régresse et les inégalités sociales se creusent. Au cours de la décennie qui a précédé 2008, non seulement la croissance a été faible mais les fruits de celle-ci n'ont bénéficié qu'aux quelques centaines de milliers de foyers les plus riches.

Cette augmentation des inégalités de revenu s'accompagne d'une précarisation d'une partie importante du salariat - spécialement les jeunes et les femmes - d'une dégradation des conditions de travail de la majorité des salariés, d'une augmentation des accidents du travail et des maladies professionnelles.

Cette dégradation peut expliquer pourquoi les manifestations ont réuni tant de monde. Mais pour aller plus loin que défiler ensemble, il ne suffit pas de partager des intérêts et des idées avec un grand nombre d'autres personnes et d'avoir conscience de cet intérêt commun. Trois conditions supplémentaires doivent être réunies : 
- une organisation capable de transformer ce mécontentement individuel en une force collective, c'est-à-dire d'abord en adhésions ;

- des caisses de grève pour permettre aux salariés de dépasser le stade de l'arrêt de travail symbolique et pour infliger aux employeurs - privés ou publics - des dommages économiques tels qu'ils ne soient pas tentés de prolonger l'épreuve de force et acceptent de négocier,

- des revendications acceptées par la majorité des adhérents et des négociateurs en capacité de discuter d'égal à égal avec les employeurs et les pouvoirs publics.

En France, à l'automne 2010, aucune de ces conditions n'est réunie. Par exemple, les syndicats n'ont pas de véritables caisses de grève et conçoivent toujours la grève comme une sorte de sacrifice auquel doivent consentir les salariés. De ce fait, les arrêts de travail sont brefs et toujours minoritaires.

\section{La situation réelle de la syndicalisation en France}

En 2003, il y avait en France, 1,7 millions de syndiqués pour 23,5 millions d'actifs salariés, soit un taux de 7\%, le plus faible de tous les pays de l'OCDE. Contrairement à ce qui est le plus souvent affirmé, ce n'est pas une fatalité historique. Entre la Libération et la fin des années 1970, 3 salariés sur 10 étaient adhérents à un syndicat et il ne s'agissait pas d'une adhésion passagère. Il y a donc bien eu une dé-syndicalisation et non une "a-syndicalisation". Il ne s'agit pas d'une méconnaissance ou d'une indifférence des salariés mais d'un véritable divorce.

En outre, cette désyndicalisation se poursuit depuis 2003 et elle continuera après les déceptions de l'automne 2010. Malgré le flou dont ils sont entourés, les effectifs officiels en témoignent. Par exemple, les chiffres de la trésorerie confédérale CGT, rendus publics lors du congrès de décembre 2009 montrait que les effectifs officiels avaient reculé de 670000 adhérents en 2002 à 630000 en 2008. En réalité, la CGT, première organisation syndicale française compte aujourd'hui à peine un demi-million de cotisants, dont moins de 400000 seraient des actifs.

De la Libération aux années 1970, il existait un maillage syndical assez dense dans les entreprises - autour des délégués et des élus - mais aussi à l'échelon local. Les cotisations étaient collectées manuellement et représentaient l'essentiel des ressources, de telle sorte que l'existence du syndicat était liée à la rencontre régulière du militant syndical avec les salariés de son entreprise et de sa branche professionnelle. Le syndicaliste qui refusait de prendre en charge les problèmes de ses adhérents était condamné à une disparition rapide. Un grand nombre de tâches étaient confiées aux simples adhérents. Les syndicats disposaient donc d'un solide enracinement social.

On pourrait parler ici d'une loi générale en science politique : l'adhésion aux associations et aux partis dépend de leur maillage organisationnel ${ }^{5}$. Plus celui-ci est dense, plus le nombre des membres est élevé et plus leur participation et leur mobilisation sont intenses. Entrent en jeu également les possibilités et les retombées de l'action collective. Plus les opportunités sont grandes, plus les élites sont accessibles, plus la participation sera dynamique et favorisera l'adhésion.

A partir de la fin des années 1970, ce maillage syndical s'est progressivement défait. Aujourd'hui la plupart des syndicalistes ont disparu des lieux de travail, même s'ils sont encore théoriquement présents dans l'entreprise et s'ils y mènent régulièrement des

\footnotetext{
${ }^{5}$ Laura Morales, Joining Political Organizations, Colchester, ECPR Press, 2009.
} 
campagnes électorales. Les salariés les rencontrent rarement, n'en reçoivent aucune aide pour résoudre leurs problèmes personnels ou collectifs. Par exemple, il est troublant de constater que les grandes entreprises, qui ont connu ces dernières années des vagues de suicides, passent pour des "bastions" syndicaux.

Partout, le syndicat s'est réduit à une poignée d'élus et mandatés, cumulant les fonctions. Il est devenu une petite institution fermée. Lorsque des conflits éclatent, les salariés préfèrent même s'organiser en dehors des syndicats et, grâce à des formes de communication transversales, ils les court-circuitent ${ }^{6}$.

Le fait que l'essentiel des ressources syndicales ne provient plus des cotisations accentue ce découplage.

\section{D'où viennent les ressources des syndicats?}

Il faut distinguer les ressources financières des ressources en nature.

Les cotisations représentent environ $15 \%$ des premières. Une proportion importante de ces cotisations provient du "recyclage" des aides que les entreprises versent à leurs syndicats. A la CGT, cela représente un timbre sur 5 encaissés par la trésorerie confédérale et un timbre sur 4 à la CFDT (les fédérations concernées étant la Poste et France Télécom, les transports, la santé, les banques et assurances, l'énergie, les métaux... $)^{7}$.

Certaines entreprises déclarent ce qu'elles versent à leurs syndicats. Par exemple, la compagnie d'assurance AXA remet un chèque syndical annuel (ou «bon de financement ») d'une cinquantaine d'euros à ses salariés. Une moitié d'entre eux le reverserait au syndicat de leur choix. Air France, Casino, EADS, France Télécom, Groupama, LCL, Renault, Safran, Thalès... effectuent des versements directs à leurs syndicats. En 2008, diverses fédérations patronales de la métallurgie ont versé au total 750000 euros à la fédération CFTC et les autres fédérations ont probablement touché des sommes équivalentes.

Les prélèvements sur les organismes de sécurité sociale et les subventions publiques représentent le reste des masses financières disponibles. Ainsi, les prélèvements sur les seuls organismes de formation permanente représentent, pour les confédérations, des sommes supérieures aux cotisations. Naturellement, ces sommes n'apparaissent pas dans les comptes officiels mais dans ceux, non publiés, de filiales comme les organismes de formation syndicale.

Quant aux aides publiques, on dispose enfin d'une première estimation grâce à une enquête administrative dans les départements du Rhône et du Loiret ${ }^{8}$. Au niveau local, le coût des décharges de service et des aides aux syndicats représenterait environ 250 euros par agent. Les auteurs indiquent qu'il s'agit d'un minimum étant donné les lacunes de l'enquête dans certains services (rectorat, police, douanes...) et des réponses très partielles de certains autres. Si l'on s'en tient à cette première indication chiffrée et qu'on l'extrapole à toute la fonction publique, cela représenterait un total de 1,319

\footnotetext{
${ }^{6}$ Jean-Pierre Basilien et al., "Découplages : le social sous contrainte", Note de conjoncture sociale, Entreprise \& Personnel, $\mathrm{n}^{\circ}$ 292, octobre 2010, p. 19-20.

${ }^{7}$ Dominique Andolfatto, Dominique Labbé, Les syndiqués en France. Qui, combien, où ?, RueilMalmaison, Liaisons, 2007.

${ }^{8}$ Corinne Desforges et al. Rapport sur le bilan des moyens alloués aux organisations syndicales dans la fonction publique. Analyse détaillée dans deux départements : le Rhône et le Loiret, Inspection générale de l'administration et al., juin 2010 (consultable en ligne sur le site de La documentation française).
} 
milliard d'euros alloués aux diverses organisations syndicales. Cela signifie que - pour les 3 fonctions publiques seules - on se situerait bien au-delà de toutes les estimations antérieures. Par exemple, le magazine Capital, dans une enquête de décembre 2007, avait estimé que la totalité des ressources syndicales - secteurs public et privé représentait 1 milliard d'euros.

En outre, ce total n'inclut pas les subventions nationales. A ce sujet, le rapport se contente d'indiquer: "Il aurait été intéressant de mesurer quelle part des subventions nationales utilisent les syndicats des deux départements considérés (...) Cependant, les représentants syndicaux rencontrés n'ont pas été en mesure de fournir des données sur ce point" (p. 23).

Deuxièmement, les ressources en nature sont essentiellement constituées de locaux et de personnels mis à disposition. Ces personnels consacrent tout ou partie de leur temps de travail au syndicat tout en continuant à être payé par leur employeur et en poursuivant leur carrière. Pour les trois fonctions publiques, le rapport déjà mentionné indique que " $0.25 \%$ des effectifs sont consacrés à l'activité syndicale" (et là encore, il s'agit d'un minimum). Les trois fonctions publiques comptant officiellement 5277000 agents (fin 2008), cela permet d'estimer le nombre minimal des «mis à disposition » dans la France entière à environ 13200 fonctionnaires. Ailleurs le rapport indique que le total des décharges de service dans les 2 départements représente 91500 journées de travail, soit quelque 416 postes «équivalents temps plein" (pour 144000 fonctionnaires dans les deux départements). Si l'on extrapole ce chiffre aux trois fonctions publiques, cela représente donc l'équivalent de 15240 postes mis à disposition.

Si l'on y ajoute les grandes écoles, la recherche publique, la banque de France, les administrations centrales, les grandes entreprises nationales - transports publics, énergie - les divers organismes de sécurité sociale, c'est, au bas mot, l'équivalent de 30000 équivalents temps pleins qui sont mis à disposition des organisations syndicales.

Ces chiffres sont des planchers et ne peuvent prendre en compte tous les "arrangements" occultes. Par exemple, le $1^{\mathrm{er}}$ novembre 2010, le syndicat SUD a révélé les conclusions d'un "audit" confidentiel interne à la RATP : 28 agents seraient mis à disposition des confédérations CGT, CFDT, UNSA, FO et CFTC ; dans les institutions représentatives du personnel, les trois quarts des heures seraient détournées au profit des syndicats; le salaire de 21 syndicalistes détachés au Comité d'Etablissement serait imputé sur le budget des activités sociales et culturelles du CE...

Enfin ces chiffrages ne tiennent pas compte de détachements de personnels également des syndicalistes - auprès de certains organismes à caractère social. La Chambre régionale des comptes d'Ile-de-France épinglait, en octobre 2010, le financement par la mairie de Paris de postes de "correspondants sociaux" au profit d'une mutuelle du personnel à hauteur de plus d'un million d'euros. Cela représenterait de l'ordre de 20 à 30 postes. La ville de Paris - évoquant un dispositif qui remonte à 1948 et en voie d'extinction - a admis l'existence de 5 à 7 postes.

Avant 2008, la plupart de ces aides étaient illégales. La loi de 2008, réformant la représentativité syndicale, en a légalisé une bonne partie mais, en contrepartie, elle avait posé le principe d'une "transparence" de ces ressources. Les décrets d'application, parus en décembre 2009, privent cette disposition de toute portée. Aucune sanction n'est prévue en cas de non-publication. Les organismes satellites à caractère commercial, où sont logées certaines recettes sensibles, ne sont pas inclus dans le périmètre soumis à publication. Surtout, la principale ressource des syndicats (les mises à disposition de personnel) n'est pas incluse dans les chiffres à publier. C'est finalement comme si le 
budget de l'Etat était présenté en oubliant la TVA et l'essentiel des postes de fonctionnaires...

\section{Quelles sont les conséquences de cette situation et comment en sortir?}

D'abord, le syndicaliste français ne dispose plus - et n'a plus véritablement besoin d'implantations sociales. A part quelques contacts superficiels, tous les 3 ou 4 ans, lors des campagnes électorales, il paraît coupé du milieu professionnel qu'il est censé représenter. La "carrière syndicale" se déroule dans des appareils clos, distants des lieux de travail et de la société française.

Cette situation entraîne une double dépendance des syndicalistes. D'une part, pour sa carrière, le syndicaliste dépend de son employeur, c'est-à-dire, en pratique, du service des "ressources humaines" - ou de son chef de service - avec qui il négocie et envers lequel il a intérêt à se montrer conciliant. D'autre part, la direction du syndicat établit la liste des "mandatés" et des "mis à disposition" et met fin à leurs fonctions. Le syndicaliste doit donc veiller à satisfaire les directions syndicales qui sont naturellement très attentives aux ressources institutionnelles mentionnées ci-dessus et à leur pérennité. Le syndicaliste prêtera donc une oreille attentive à la direction de son entreprise - ou de son administration - et à celle de son syndicat.

Bien sûr, il se soucie aussi de ne pas perdre trop de voix et il met pour cela en scène un dialogue social difficile, avec la complicité tacite de l'employeur. C'est à un brouillage de ce genre que se sont livrées les directions syndicales pendant l'automne 2010. Dans le même temps qu'elles vilipendaient l'Etat et prétendaient que tout contact était rompu avec lui, ces mêmes directions négociaient un accord "cadre" sur le dialogue social dans la fonction publique, accord qui comporte un chapitre sur le renforcement des droits syndicaux et sur la carrière des syndicalistes de la fonction publique.

Plus au fond, en 2008, le MEDEF s'est mis d'accord avec la CFDT et la CGT pour établir une sorte de partenariat stratégique. Celui-ci s'est concrétisé dans la réforme de la représentativité syndicale. Il s'agit de renforcer la position dominante de ces deux confédérations - en élevant des barrières contre les dissidents et les contestataires - et de légaliser le financement des syndicats par les employeurs. Pour le patronat, ce partenariat doit permettre d'achever une révolution silencieuse grâce à la négociation collective d'entreprise.

En effet, le tableau d'ensemble de la négociation collective dans le secteur privé dressé annuellement par le ministère du Travail - montre une progression continue du nombre des entreprises et des salariés concernés, mais aussi une ampleur croissante des thèmes abordés et de la portée de ces textes : temps de travail, individualisation des rémunérations et des carrières, flexibilité, épargne salariale...

Les rapports indiquent également que, dans $90 \%$ des établissements où s'ouvre une négociation, on aboutit à un accord et que les syndicats, parties à la négociation, signent entre $91 \%$ (CFDT) et $83 \%$ de ces textes (CGT). Ces scores ne doivent pas étonner. A tous les niveaux, le patronat organise les réunions, fixe l'ordre du jour et l'agenda, tient le secrétariat, rédige les textes... Ces accords comportent souvent un chapitre particulier consacré aux moyens accordés aux syndicats. En contrepartie, ils conduisent à une remise en cause progressive et silencieuse du droit du travail, grâce à des clauses dérogatoires - notamment sur le temps de travail et l'individualisation des rémunérations -, clauses qui ne sont jamais recensées et rarement étudiées. 
L'image d'opposition systématique entre les partenaires sociaux n'a donc plus rien à voir avec la réalité des relations professionnelles en France, mais elle fournit un paravent commode aux employeurs comme aux syndicalistes.

Dans les milieux syndicaux, on parle de "mercato" pour désigner les nombreux passages d'élus ou de mandatés d'une organisation à une autre. Cette métaphore sportive montre que le syndicalisme est devenu affaire de professionnels de la représentation qui privilégient des intérêts purement organisationnels, voire personnels dont les contreparties sont faciles à imaginer.

En acceptant les aides des pouvoirs publics et des entreprises, les syndicats ont aliéné leur indépendance et se sont condamnés à l'impuissance. Il semble impossible de sortir de ce cercle vicieux sans supprimer les aides et les privilèges. Cela obligerait les syndicats à se mettre au service des salariés et à leur apporter des services utiles, à constituer des caisses de grève et à former de véritables négociateurs.

Il faudrait également limiter les possibilités de dérogations au droit du travail et aux conventions collectives nationales et prévoir que les salariés concernés soient consultés avant la mise en œuvre de ces dérogations. L'interdiction du cumul des mandats est aussi une piste à explorer. Plus largement, pourquoi tolérer les dispositifs qui limitent la démocratie dans les organisations et qui favorisent l'opacité de leur fonctionnement et de leur financement? 